\title{
ERRATUM
}

Ewgeni B. Starikov $\cdot$ Lennart Nilsson

Martin Hülsmeyer

\section{A single residue exchange between two HLA-B27 alleles triggers increased peptide flexibility}

Published online: 8 May 2004

(c) EBSA 2004

\section{European Biophysics Journal (2004) s00249-004-0390}

The first author's name should read E.B. Starikov instead of E.B.Starikow.

The online version of the original article can be found at http:// dx.doi.org/10.1007/s00249-004-0390-7

E. B. Starikov $\cdot$ L. Nilsson $(\square)$

Department of Biosciences at NOVUM,

Karolinska Institutet, 14157 Huddinge, Sweden

E-mail: lennart.nilsson@biosci.ki.se

M. Hülsmeyer

Institute for Crystallography, Free University Berlin,

14195 Berlin, Germany 